\title{
In silico secretome analysis approach for next generation sequencing transcriptomic data
}

\author{
Gagan Garg ${ }^{1}$, Shoba Ranganathan ${ }^{1,2^{*}}$ \\ From Asia Pacific Bioinformatics Network (APBioNet) Tenth International Conference on Bioinformatics - First \\ ISCB Asia Joint Conference 2011 (InCoB/ISCB-Asia 2011) \\ Kuala Lumpur, Malaysia. 30 November - 2 December 2011
}

\begin{abstract}
Background: Excretory/secretory proteins (ESPs) play a major role in parasitic infection as they are present at the host-parasite interface and regulate host immune system. In case of parasitic helminths, transcriptomics has been used extensively to understand the molecular basis of parasitism and for developing novel therapeutic strategies against parasitic infections. However, none of transcriptomic studies have extensively covered ES protein prediction for identifying novel therapeutic targets, especially as parasites adopt non-classical secretion pathways.

Results: We developed a semi-automated computational approach for prediction and annotation of ES proteins using transcriptomic data from next generation sequencing platforms. For the prediction of non-classically secreted proteins, we have used an improved computational strategy, together with homology matching to a dataset of experimentally determined parasitic helminth ES proteins. We applied this protocol to analyse 454 short reads of parasitic nematode, Strongyloides ratti. From 296231 reads, we derived 28901 contigs, which were translated into 20877 proteins. Based on our improved ES protein prediction pipeline, we identified 2572 ES proteins, of which 407 (1.9\%) proteins have classical N-terminal signal peptides, 923 (4.4\%) were computationally identified as nonclassically secreted while 1516 (7.26\%) were identified by homology to experimentally identified parasitic helminth ES proteins. Out of 2572 ES proteins, 2310 (89.8\%) ES proteins had homologues in the free-living nematode Caenorhabditis elegans and 2220 (86.3\%) in parasitic nematodes. We could functionally annotate 1591 (61.8\%) ES proteins with protein families and domains and establish pathway associations for 691 (26.8\%) proteins. In addition, we have identified 19 representative ES proteins, which have no homologues in the host organism but homologous to lethal RNAi phenotypes in C. elegans, as potential therapeutic targets.

Conclusion: We report a comprehensive approach using freely available computational tools for the secretome analysis of NGS data. This approach has been applied to S. ratti 454 transcriptomic data for in silico excretory/ secretory proteins prediction and analysis, providing a foundation for developing new therapeutic solutions for parasitic infections.
\end{abstract}

\section{Background}

The secretome of an organism is defined as the subset of proteins secreted by the cell [1]. This subset of proteins is usually known as excretory/secretory (ES) proteins [2], plays an important role in producing clinical infections in the host organism. ES proteins are the

\footnotetext{
* Correspondence: shoba.ranganathan@mq.edu.au

'Dept. of Chemistry and Biomolecular Sciences, Macquarie University, Sydney NSW 2109, Australia

Full list of author information is available at the end of the article
}

choice of new therapeutic solutions for different clinical infections, especially in the case of parasitic infections $[3,4]$ because these proteins are present at the host-parasite interface and act as immunoregulators to host immune recognition for parasite survival inside the host organism [5].

Transcriptomic data is the representation of actively expressed genes in a cell at any given time. Earlier transcriptomic studies were based on generation of expressed sequence tags (ESTs) generated at different

\section{(Ciomed Central}


stages of an organism using traditional Sanger sequencing. These studies were restricted to the analysis of a few thousand ESTs at a time. Recent technological improvements in cDNA sequencing, using next generation sequencing (NGS) platforms, are able to generate millions of reads, to record the transcript profile of an organism at a given developmental stage. The read length generated through NGS is quiet short (50-400 bases) as compared to traditional Sanger sequencing (800-1000 bases). Thus, the assembly of shorter reads is challenging in terms of computational power and resources needed. These reads are assembled into long consensus sequences (clusters) known as contigs using assemblers such as ABySS [6], Velvet [7] and MIRA [8], which have been reviewed in a recent study [9]. ABySS and Velvet provide good results for genome assembly, while MIRA is very well tested for handling de novo transcriptome assembly [10]. Since the genomes of only a very few parasitic nematodes are currently available, de novo assemblers such as MIRA are the only option for NGS data from these neglected organisms.

Recently, NGS platforms have been used to generate large amounts of transcriptomic data for different organisms, including several helminth parasites like Fasciola gigantica [11], Fasciola hepatica[12], Trichostrongylus colubriformis[13], Oesophagostomum dentatum[14], Haemonchus contortus [15], Dictyocaulus viviparus[16], Necator americanus [17], Clonorchis sinensis[18], Opisthorchis viverrini [18] and Teladorsagia circumcinta [19]. Here, NGS data has been assembled with CAP3 alone $[14,16]$ or with MIRA followed by CAP3 $[12,18]$, based on combinations of assemblers performing better in a recent study [10]. However, none of these studies have extensively covered ES protein prediction and further analysis, for identifying therapeutic targets.

ES proteins were once considered to be secreted only through conventional secretion pathways, using N-terminal signal peptide signatures, but there are now many proteins which are found to be secreted by non-classical secretory pathways [20]. Usually non-classical secretory proteins are predicted through SecretomeP [21], which is the most widely used tool for non-classical secretory proteins. However in case of parasites, SecretomeP is not able to completely predict non-classical secretory proteins, as shown in the study of Brugia malayi[22]. Hence, a novel approach to identifying non-classically secreted proteins is required for comprehensive secretome analysis.

Transcriptomic data has been used extensively for the prediction of ES proteins in parasitic helminth studies [23]. EST2Secretome, a computational prediction and annotation pipeline for ES proteins from our group, was designed to handle ESTs from Sanger sequencing and currently has the following limitations: (i) assembly of short reads, (ii) prediction of non-classical secretory proteins and (iii) pathway mapping using KOBAS $[24,25]$, which contains pathways that are not regularly updated.

In the present study, we have developed an updated computational approach for the prediction and annotation of ES proteins using NGS transcriptomic data overcoming the limitations of the earlier EST2Secretome pipeline. We have developed a robust assembly protocol for NGS data. In order to identify non-classically secreted proteins that are missed by SecretomeP, we have also compiled a dataset of experimentally determined ES proteins of parasitic helminths for homologybased prediction (details in the Methods section). Additionally, we have replaced KOBAS with KAAS [26], for efficient and up-to-date pathway identification.

We applied our approach to $\sim 0.3 \mathrm{M} 454$ transcriptomic reads for a parasitic nematode, Strongyloides ratti, which is a gastro intestinal nematode that infects rats, comprehensively reviewed by Viney [27] and is a Clade IV parasite [28]. Genome data is available only for the free living nematodes, C. elegans[29] and C. briggsae[30] from Clade V, which is adjacent to Clade IV and for a parasite, Brugia malayi[31] from Clade III, which is not similar to Clade IV parasites, whereas limited transcriptomic and proteomic data from experimental studies are available for several helminth parasites. As such, a BLASTX against a reference organism, as proposed recently [32] will not provide comprehensive annotation results, unless the fully annotated proteome of a very similar organism is available.

In adult phase, $S$. ratti is present in both parasitic (females only) and free living forms (male and female) [27]. Eggs produced by parasitic females develop into free living males, free living females and parasitic females by different larval stages. Our dataset is derived from the adult nematode, which includes parasitic and free living forms (sequencing details in the Methods section). The NGS data has been clustered and translated into proteins and ES proteins predicted using a series of computational tools, augmented by homology matching to our in-house dataset of experimentally determined parasitic helminth ES proteins. Predicted ES proteins have been annotated functionally in terms of protein families, domains and biochemical pathways. ES proteins have also been compared with proteomic data of the host (rat) and other nematodes, with an emphasis on the best characterized nematode, C. elegans. Such annotation techniques have enabled us to identify 19 novel targets, matching to lethal RNAi phenotypes in C. elegans, which could be considered in the development of future therapeutic strategies. 


\section{Methods}

\section{cDNA sequencing data sets}

For this study, S. ratti cDNA sequencing data from the University of Liverpool [33] is used. cDNA libraries were prepared from adult helminths, comprising a mixture of parasitic females, free-living males and free-living females. Sequencing was performed using 454-FLX platform (Roche diagnostics). The pyrosequencing procedure used to prepare this dataset is described elsewhere [34].

\section{Components of computational approach}

Our approach to predict and annotate ES proteins is divided into three phases, shown in Figure 1, corresponding approximately to those in EST2Secretome [23]. EST2Secretome was developed with the aim to predict and annotate ES proteins from ESTs (generated mainly using Sanger sequencing) mainly from parasitic nematodes. Now with the use of NGS, the input sequence data has changed considerably in terms of read length and number; necessitating modifications to tackle NGS data as well reliably predict non-classical protein secretion and use updated annotation tools.

\section{Phase I: extraction and assembly of data}

FASTA and associated quality files were extracted from SFF file along with clipping of sequence adapters using the sff_extract software [35]. Extracted data from sff files is first assembled using the MIRA [8] (V3.2.0rc1) assembler using quality information. MIRA is our preferred assembler as it is an open source tool which is considered reliable for data from different NGS platforms [8] and it

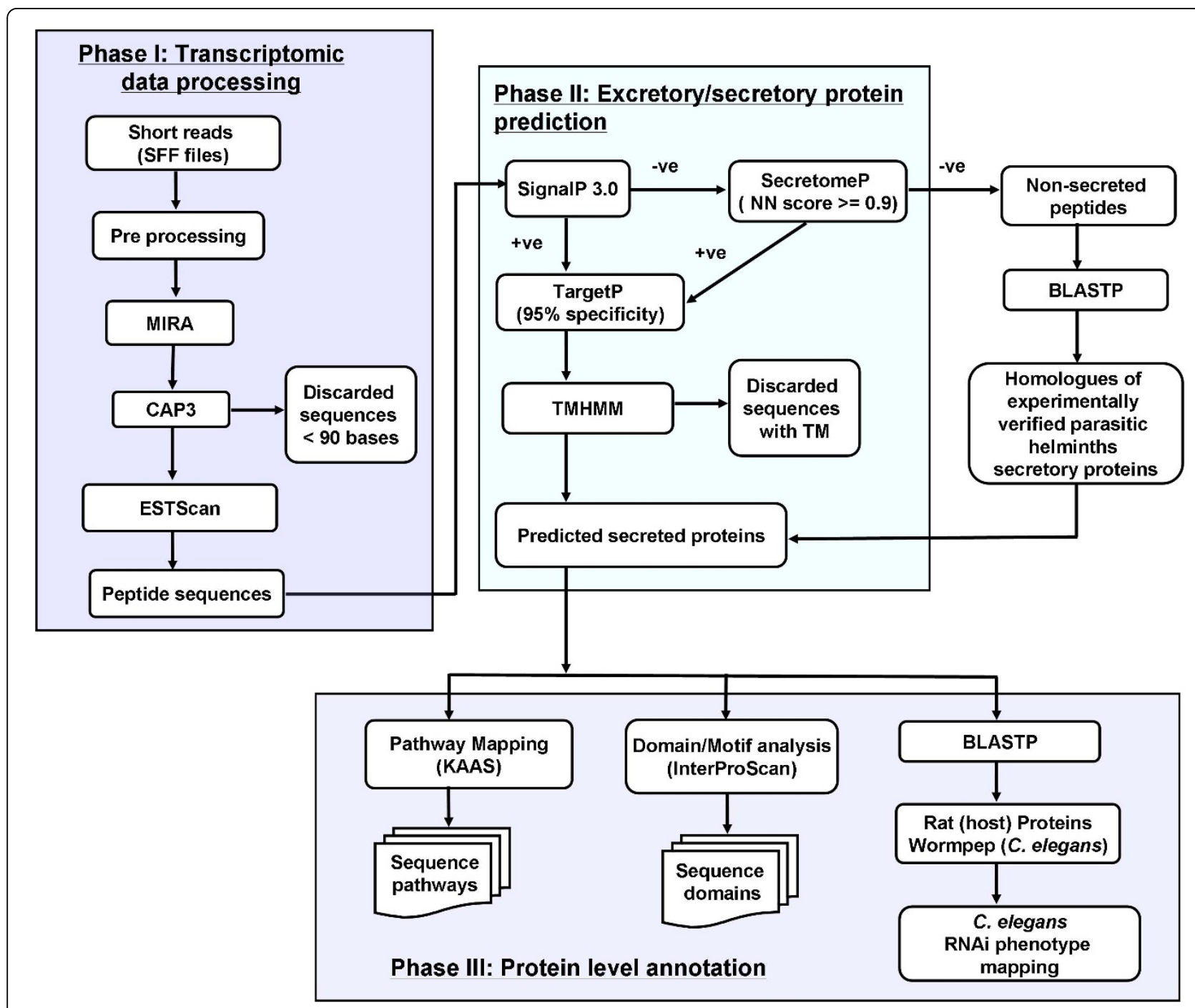

Figure 1 Bioinformatics workflow for secretome analysis. Bioinformatics workflow comprising Phase I (pre-processing and assembly), II (prediction of excretory/secretory proteins) and III (Protein-level annotation) were augmented by homologue identification from nematodes as well as parasitic nematodes, using specialized databases. 
has been very well tested in other parasitic helminth transcriptomic studies [12,18]. For this dataset, we have used MIRA, ABYSS and Velvet, compared with Newbler (data not shown), MIRA giving the longest contigs. Contigs generated by MIRA are further passed to the Contig Assembly Program (CAP3) [36], to extend the MIRA assembly. This is in accord with an earlier study which suggests that serial assembly from two assemblers can improve the quality of the assembly [10]. Second order contigs generated using CAP3 are combined with MIRA contigs, to be conceptually translated into putative proteins using ESTScan [37].

\section{Phase II: prediction of excretory secretory proteins}

ES proteins were predicted using a combination of four tools, SecretomeP [21], SignalP [38], TargetP [39] and TMHMM [40]. SignalP is used for the prediction of classical secretory proteins, while SecretomeP predicts non-classical secretory proteins. TargetP is for the prediction of mitochondrial proteins and TMHMM identifies transmembrane proteins. Firstly, the proteins generated from ESTScan are passed to SignalP for prediction of classical secreted proteins. All the proteins, which are predicted as non-secretory (proteins having D score and signal peptide probability less than 0.5 ) are then passed to SecretomeP for prediction of non-classical secretory proteins. Proteins which obtain neural network (NN) score of greater than or equal to 0.9 are considered as non-classical secretory proteins. All the classical and non-classical secretory proteins are merged together and then scanned by TargetP. Proteins predicted as mitochondrial proteins by TargetP are omitted out from the set of predicted ES proteins and passed to TMHMM. Finally the proteins which are predicted to have no transmembrane helices are considered as ES proteins.

In addition to standard computational approaches for the prediction of ES proteins, we compiled a list of 1080 ES protein sequences of parasitic helminths (Brugia malayi, Teladorsagia circumcinta, Schistosoma mansoni, Ancylostoma caninum, Schistosoma japonicum, Clonorchis sinesis and Fasciola hepatica) from the literature [22,41-49]. A homology-based search with BLASTP [50] is used to further extract ES proteins from proteins which are predicted to be non-secretory by SecretomeP.

The results from computational tools are combined with those from BLAST searches, for functional annotation and analysis in Phase III.

\section{Phase III: annotation and comparative analysis of ES proteins}

All the predicted ES proteins are annotated using a number of tools. We used Interproscan [51] for protein domain and family classification. KAAS [26] is used for mapping ES proteins to KEGG pathways and to KEGG BRITE objects [52-54]. ES proteins are searched for sequence similarity against the Wormpep database (WS224) [55] for proteins similar to C. elegans. ES proteins are also searched for sequence similarity against rat (host) proteins and parasitic nematodes using BLASTP algorithm, to identify parasite-specific proteins. Comparative analysis of similarity of ES proteins with rat, parasitic nematodes and C. elegans proteins are analyzed using Simitri [56]. Proteins not homologous to the host (rat) proteome are further screened for RNAi phenotypes in C. elegans.

\section{Hardware specifications}

All the programs used in this study were installed on a 16 CPU Linux cluster (2.4 GHz, Intel(R)Xeon(R) E5530, 32 RAM) running on ubuntu server operating system. The computer intensive steps are sequence assembly (MIRA, CAP3) and protein functional annotation mapping (Interproscan). All other programs will run efficiently on current desktop systems.

\section{Results}

A semi-automated computational approach, incorporating three key components, was constructed. The different components of the workflow system (Figure 1) are linked using Perl, Python and bash shell scripts. This approach was applied to $S$. ratti 454 transcriptomic dataset to show its efficacy and utility.

\section{Extraction and assembly of $S$. ratti data sets}

Initially 296231 short reads (69488625 bases) were extracted from the sff file with $234 \pm 62$ bases (average length \pm standard deviation), and a GC content of $39.7 \%$. The de novo assembly from MIRA results in 33222 contigs, which were passed to CAP3 to get a more robust assembly, with a minimum sequence overlap length of 40 bases and an identity threshold of $90 \%$. Using CAP3, we are able to achieve a maximum contig length of 3620 bases as compared to maximum contig length of 2607 bases by Newbler [34]. The CAP3 assembly results in 3056 second order contigs and 25845 MIRA contigs (not assembled further by CAP3). The difference in results using MIRA+CAP3 and Newbler are shown in Table 1. We consider 25765 (99.6\%) contigs with a minimum length of 90 bases, discarding sequences yielding peptides $<30$ amino acids, for further secretory protein prediction and analyses. A total of 3056 second order contigs and 25765 contigs were conceptually translated into 20877 proteins by ESTScan.

\section{Prediction of ES proteins}

ES protein prediction is carried out in Phase II of the pipeline (Figure 1). Firstly, 407 (1.9\%) proteins were predicted as classical secreted proteins using SignalP. The remaining 20470 (98.05\%) proteins, which were 
Table 1 Comparison of results from different NGS assemblers

\begin{tabular}{llllllll}
\hline Assembler & No. of second order contigs & No. of contigs & Largest contig & Average length & N50* & N90* & Number of bases \\
\hline MIRA [8] + CAP3 [29] & 3056 & 25845 & 3620 & 402.36 & 406 & 253 & 11628536 \\
Newbler [26] & & 25127 & 2607 & 407.11 & 409 & 252 & 10229510 \\
\hline
\end{tabular}

*N50 refers to the length of the shortest contig such that the sum of contigs of equal length or longer is at least $50 \%$ of the total assembly size. While N90 refers to the length of the shortest contig such that the sum of contigs of equal length or longer is at least $90 \%$ of the total assembly size.

predicted as non secretory by SignalP were processed by SecretomeP for prediction of non-classical secretory proteins. A total of $923(4.4 \%)$ proteins were predicted as non-classical secretory proteins using SecretomeP. The classical and non-classical secretory proteins (1330, 6.3\%) from these two programs were analyzed by TargetP for mitochondrial proteins. Only 18 proteins were predicted as mitochondrial proteins using TargetP at 95\% specificity. These 18 proteins were removed from the set of 1330 secreted proteins while 1312 secretory proteins were passed to TMHMM for the prediction of transmembrane proteins. 256 proteins, predicted as transmembrane proteins having one or more transmembrane helices, were removed from the secretory protein dataset. A total of 1056 (5.05\%) proteins were finally predicted as ES proteins from the computational prediction pipeline.

Proteins that were considered non-secretory by SecretomeP were matched to our in-house dataset of 1080 non redundant experimentally determined parasitic helminth proteins, using the BLASTP similarity search. We found an additional 1516 (7.26\%) proteins similar to known ES proteins by this homology search approach. Thus, for annotation and analyses in Phase III, we compiled a total of 2572 ES proteins, which is $12.3 \%$ of our putative proteins. This dataset is a more comprehensive collection of ES proteins of $S$. ratti, compared to those reported by other $S$. ratti secretome studies $[57,58]$.

\section{Annotation of S. ratti ES proteins}

ES proteins are annotated based on protein families and domains using Interproscan and mapped to biochemical pathways using KAAS. Out of 2572 ES proteins predicted, we were able to annotate 1591 (61.8\%) proteins with protein domains and families. The most represented Interpro terms are shown in Table 2 (complete results available from Additional file 1). We established pathway associations to 691 (26.8\%) ES proteins. Among the most represented pathways are metabolic pathways, which are important for parasite survival inside the host. Predicted ES proteins are associated with important biological molecules, like enzymes, peptidases and protein kinases. The most represented KEGG BRITE objects and KEGG pathways are shown in Table 3 (full annotation available from Additional file 2) and Table 4 (full annotation available from Additional file 3).

\section{Comparative analysis of S. ratti ES proteins with other organisms}

2310 (89.8\%) S. ratti ES proteins had homologues in the free-living nematode, C. elegans. 2220 (86.3\%) ES proteins had homologues in parasitic nematodes. As $S$. ratti infects rats, we checked the similarity of ES proteins with the rat proteome. Similarity of $S$. ratti ES proteins to $C$. elegans, parasitic nematodes and rat proteins is shown using Simitri in Figure 2. We found 537 (20.8\%) ES proteins had no homologues present in rat and are therefore preferred targets for parasite intervention strategies. 142 ES proteins are novel in the $S$. ratti dataset, with no known homologues to the host or any other nematode. 233 (9\%) ES proteins, which are not present in the host (rat), have homologues present in C. elegans. Of these, 19 ES proteins (predicted from second order contigs from CAP3 assembly), which have lethal RNAi phenotypes present in C. elegans, (complete RNAi phenotype mapping available from Additional file 4) and represent potential therapeutic targets (Additional file 5).

\section{Discussion}

We demonstrated the utility of our new computational approach for the comprehensive prediction and analysis

Table 2 Top 15 most represented protein domains found in ES proteins using Interproscan

\begin{tabular}{lll}
\hline InterPro description & $\begin{array}{l}\text { InterPro } \\
\text { code }\end{array}$ & $\begin{array}{l}\text { Number of ES } \\
\text { proteins (\%) }\end{array}$ \\
\hline Protein Kinase like domain & IPR011009 & $126(4.90)$ \\
Protein kinase, catalytic domain & IPR000719 & $114(4.43)$ \\
Serine/threonine-protein kinase like & IPR017442 & $99(3.85)$ \\
domain & & \\
Serine/threonine-protein kinase domain & IPR002290 & $64(2.49)$ \\
Serine/threonine-protein kinase active site & IPR008271 & $52(2.02)$ \\
WD40 repeat like domain & IPR011046 & $40(1.55)$ \\
WD40 repeat subgroup & IPR019781 & $39(1.52)$ \\
WD40/YVTN repeat like domain & IPR015943 & $39(1.52)$ \\
WD40 repeat & IPR001680 & $39(1.52)$ \\
WD40 repeat domain & IPR017986 & $38(1.47)$ \\
Tyrosine-protein kinase catalytic domain & IPR020635 & $37(1.44)$ \\
WD40 repeat 2 & IPR019782 & $37(1.44)$ \\
Helicase C & IPR001650 & $35(1.36)$ \\
NAD(P)-binding domain & IPR016040 & $29(1.13)$ \\
Immunoglobulin-like fold & IPR013783 & $28(1.09)$ \\
\hline
\end{tabular}


Table 3 Top 15 most represented KEGG pathways found in ES proteins predicted by KAAS

\begin{tabular}{ll}
\hline Pathway name & $\begin{array}{l}\text { Number of ES proteins } \\
\text { represented (\%) }\end{array}$ \\
\hline Metabolic pathways & $109(4.24)$ \\
Protein processing in endoplasmic & $57(2.22)$ \\
reticulum & \\
Ubiquitin mediated proteolysis & $44(1.71)$ \\
Wnt signalling pathway & $29(1.13)$ \\
Glycolysis / Gluconeogenesis & $28(1.08)$ \\
Spliceosome & $28(1.08)$ \\
Glutathione metabolism & $26(1.01)$ \\
Circadian rhythm - mammal & $22(0.85)$ \\
TGF- beta signalling pathway & $22(0.85)$ \\
RNA transport & $20(0.77)$ \\
Endocytosis & $20(0.77)$ \\
Purine metabolism & $19(0.74)$ \\
Phagosome & $19(0.74)$ \\
Proteasome & $18(0.70)$ \\
Drug metabolism & $17(0.66)$ \\
\hline
\end{tabular}

of ES proteins from transcriptomic data generated by NGS. The protocol will be implemented in a web server, in the future, after extensive testing of different assembly programs, and considering the choice of specific assemblers, based on the transcriptomic dataset, as proposed by Kumar and Blaxter [10]. For this study, we have selected programs that are freely available under academic licence. All the programs used in our approach are available with free academic licence, which can be easily installed on Linux platforms. Our use of MIRA followed by CAP3 for assembly of NGS data is

Table 4 Top 15 most represented KEGG BRITE objects found in ES proteins predicted by KAAS

\begin{tabular}{ll}
\hline BRITE object & $\begin{array}{l}\text { Number of ES proteins } \\
\text { represented (\%) }\end{array}$ \\
\hline Enzymes & $282(10.96)$ \\
Spliceosome & $49(1.90)$ \\
Chaperons and folding catalysts & $44(1.71)$ \\
Peptidases & $44(1.71)$ \\
Protein kinases & $43(1.67)$ \\
Ubiquitin system & $37(1.44)$ \\
Chromosome & $34(1.32)$ \\
Cytoskeleton proteins & $27(1.05)$ \\
DNA repair and recombination & $21(0.82)$ \\
proteins & $19(0.74)$ \\
GTP-binding proteins & $18(0.70)$ \\
Proteasome & $17(0.66)$ \\
Transcription factors & $16(0.62)$ \\
Ribosome biogenesis & $11(0.43)$ \\
Translation factors & $9(0.35)$ \\
DNA replication proteins &
\end{tabular}

simpler than the assembler combinations proposed by Kumar and Blaxter [10] and also used by studies on Fasciola hepatica [12], Clonorchis sinensis [18] and Opisthorchis viverrini[18] to generate second order contigs by CAP3 from contigs generated by MIRA which have open reading frames. The whole assembly for the current dataset was performed in approximately 3 hours CPU time using both MIRA and CAP3, whereas the use of CAP3 alone was not possible due to memory overflow with the current dataset, using hardware specified in the methods section. Although all the studies discussed here are more comprehensive in terms of transcriptome coverage (more than $0.5 \mathrm{M} 454$ reads were generated), which is higher as compared to our current dataset of $\sim 0.3 \mathrm{M}$, none of them have comprehensively studied ES proteins. For example, the 454 transcriptomic study on Fasciola hepatica [12] reported only 1812 ES proteins (only 4\%) from 44597 putative protein sequences generated from ESTScan, followed by ES protein predictions based on signal peptide identification by SignalP.

\section{Biological implications of the results}

Millions of people globally suffer from Strongyloidiasis, caused by the parasitic nematode, Strongyloides stercoralis. S. ratti is a common gastro-intestinal parasite of the rat, which is used as a model to study Strongyloidiasis. Here, we have analysed S. ratti transcriptomic data from parasitic females, free-living males and free-living females for the prediction and analysis of ES proteins. Of the dataset of 2572 ES proteins 2310 (89.8\%) had homologues in the free-living nematode, C. elegans, which is similar to earlier reported findings in Strongyloides EST analysis studies [59]. Many predicted ES proteins map to protein kinase domains as shown in Table 2 , which are reported to be essential for parasitic activity in parasitic nematodes [60]. Protein kinases play a central role in signal transduction and hence are considered as drugabble targets. Another representative Interpro protein domains among $S$. ratti ES proteins were WD40 repeat domains $(7.5 \%)$, which are associated with signalling transduction pathways [61]. These domains were also found among the top 20 most represented Interpro protein domains of $O$. dentatum putative proteins [14]. ES proteins also map to ribosomal protein interpro domains such as IPR000589 (Ribosomal protein S15), which is associated with ageing in S. ratti [62]. All the most representative KEGG pathways mapped to ES proteins shown in table 3 are required for parasite survival inside the host, as the secretome of a parasite is representative of its genome in the host environment. Major ES proteins map to enzymes, which are essential for metabolic pathways functioning and also very well reflected in our protein domain mapping. Other KEGG 


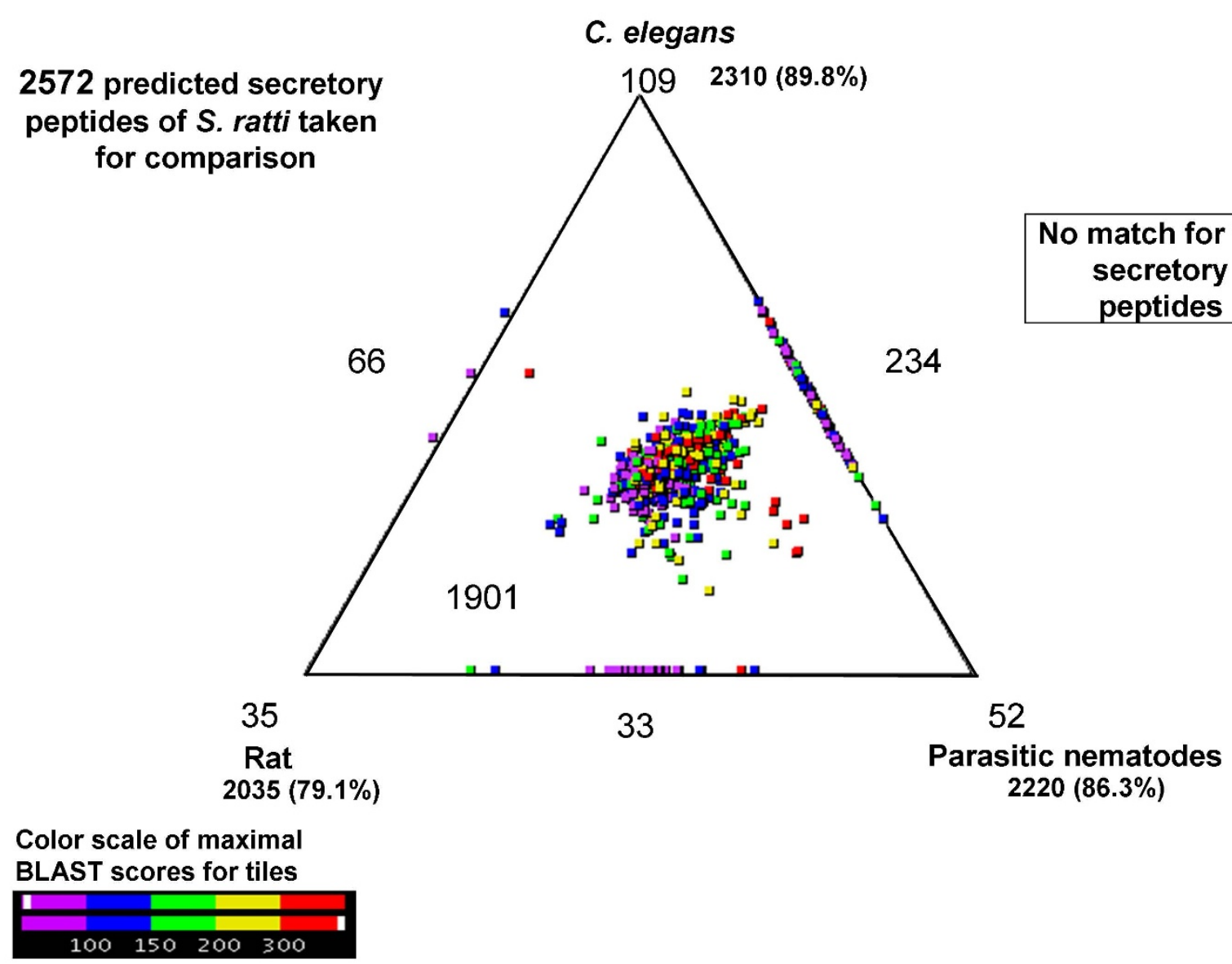

Figure 2 Comparison of S. ratti ES proteins with C. elegans, parasitic nematodes and rat (host) protein sequence databases using SimiTri. The numbers at each vertex indicate the number of proteins matching only that specific database. The numbers on the edges indicate the number of proteins matching the two databases linked by that edge. The number within the triangle indicates the number of S. ratti ES proteins with matches to all three databases.

pathways like purine metabolism and glutathione metabolism found in this study were also found in other parasitic nematodes excretory/secretory proteins analysis [23]. $22(0.85 \%)$ ES proteins were mapped to the circadian rhythm - mammal pathway in C. elegans. This pathway is unexpected in the case of ES proteins of nematodes, however three proteins S-phase kinase-associated protein 1 (KO3094), cullin 1 (KO3347) and F-box and WD-40 domain protein 1/11 (KO3362) which were found in our ES proteins are common to Ubiquitin mediated proteolysis in C. elegans. The common components of several pathways have led to this unexpected result. KEGG BRITE objects (representative objects shown in Table 4) reflect the presence of essential proteins such as protein kinases, peptidases and proteasome among ES proteins for S. ratti survival inside the host organism. 44 (1.71\%) ES proteins map to chaperones, which are responsible for host immune system modulation, such as the recently characterised $S$. ratti heat shock protein 10 [63]. Along with well known protein families found in ES proteins, we found some protein categories such as chromosome, DNA replication proteins and DNA repair and recombination proteins which are expected to be localized in the nucleus but found in S. ratti ES proteins. This pattern of exporting nuclear proteins to the secretome of a parasitic nematode was also observed in Meloidogyne incognita [64]. 66 secreted proteins were identified with putative nuclear localization such as DNA and RNA binding proteins including helicases in $M$. incognita, of which we observed the presence of helicase $C$ domain in 35 (1.36\%) S. ratti ES proteins. Contig 1289 and Contig 428 map to the metalloproteinase precursor in S. stercoralis [65], this is also well characterized protein in Trichinella spirallis[66]. Expresssion of an S. stercoralis metalloproteinase homologue was also found in the recent 
transcript analysis of another intestinal nematode, Strongyloides venezuelensis [67]. Many of these potential therapeutic targets map to hypothetical proteins present in C. elegans, C. briggsae and B. malayi and having lethal phenotypes according to C. elegans RNAi phenotype mapping and could be considered as parasitism central genes [68] of $S$. ratti. Many of the putative proteins from $S$. ratti could be examined further after the publication of $S$. ratti genome, which is expected soon [69].

\section{Methodological limitations}

Integrated approaches similar to the one discussed in this paper have been applied to several socio-economically important parasites. These approaches are based on data available on the reference organism of that taxonomic order where limited data is available for the subject organism. For example, C. elegans is the most studied organism among nematodes. C. elegans data was used to create the translation matrix used by ESTScan, to translate potential coding regions in the assembled contigs into protein sequences. These translated coding regions were then used for ES proteins prediction. The use of a reference organism data for the translation matrix instead of using actual organism information may lead to false positives in peptides prediction as well as in ES protein prediction. Another limiting factor is that we are looking into the annotation of protein function in terms of primary sequence alone, rather than the 3D structure. Therefore, all the therapeutic targets predicted in this study are preliminary predictions which need to be further validated by additional computation analysis such as structural modelling and by experimental assays.

\section{Conclusions}

In this paper we demonstrate how different computational tools can be used together to extract the useful information of ES proteins from transcriptomic data. All the programs used in our approach are open source tools that are freely available for academic purposes. With the advent of NGS technologies, while there is a massive increase in sequence data, this data is extremely fragmented and of no use for information extraction as output from the sequencer. Our methodology will help in rapid assembly, fast annotation and reliable prediction of ES proteins. The approach is a generalized method which can be applied to any organism, although its main application is for neglected organisms whose genomes are not yet sequenced, with limited functional knowledge. Although we have used 454 transcriptomic data in this study but this methodology can be applied to transcriptomic data from other NGS platforms with slight modifications in terms of pre-processing, as data output formats obtained from different NGS platforms are different. Thus, this system will help us to carry out secretome studies for other parasitic organisms in future.

\section{Additional material}

Additional file 1: Protein domain mapping of S. ratti ES proteins
Represented Interpro domains found in S. ratti ES proteins using
Interproscan (sheet1). Protein domains mapping of S. ratti excretory/
secretory proteins (sheet2).

Additional file 2: KEGG pathways mapping of S. ratti ES proteins. Represented KEGG pathways found in ES proteins predicted by KAAS (Table S2).

Additional file 3: KEGG BRITE objects mapping of S. ratti ES proteins. Represented KEGG BRITE objects found in ES proteins predicted by KAAS (Table S3).

Additional file 4: RNAi Phenotype mapping of S. ratti ES proteins. RNAi Phenotype mapping of S. ratti ES proteins against known C. elegans known phenotypes (sheet1).

Additional file 5: Representative therapeutic targets set of S. ratti ES proteins. Representative therapeutic targets set of S. ratti ES proteins, homologous to $C$. elegans proteins with lethal RNAi phenotype and with no homologue in the host, rat.

List of abbreviations used

BRITE: Biomolecular Relations in Information Transmission and Expression; KEGG: Kyoto Encyclopedia of Genes and Genomes; KAAS: KEGG automatic annotation server.

\section{Acknowledgements}

We would like to thank Dr. Steve Paterson for providing the Strongyloides ratti cDNA sequencing data. We are thankful to Prof. Minoru Kanehisa for providing us the stand alone copy of KAAS program. GG would like to acknowledge Macquarie University for an Australian Post-graduate Award scholarship.

This article has been published as part of BMC Genomics Volume 12 Supplement 3, 2011: Tenth International Conference on Bioinformatics - First ISCB Asia Joint Conference 2011 (InCoB/ISCB-Asia 2011): Computational Biology. The full contents of the supplement are available online at http:// www.biomedcentral.com/1471-2164/12?issue $=$ S3.

\section{Author details}

${ }^{1}$ Dept. of Chemistry and Biomolecular Sciences, Macquarie University, Sydney NSW 2109, Australia. ${ }^{2}$ Dept. of Biochemistry, Yong Loo Lin School of Medicine, National University of Singapore, 8 Medical Drive, Singapore 117597.

\section{Authors' contributions}

SR directed the study. GG did the analysis. SR and GG contributed to writing the manuscript.

\section{Competing interests}

The authors declare that they have no competing interests.

Published: 30 November 2011

\section{References}

1. Skach WR: The expanding role of the ER translocon in membrane protein folding. J Cell Biol 2007, 179:1333-5.

2. Tjalsma H, Bolhuis A, Jongbloed JD, Bron S, van Dijl JM: Signal peptidedependent protein transport in Bacillus subtilis: a genome-based survey of the secretome. Microbiol Mol Biol Rev 2000, 64:515-547.

3. Bonin-Debs AL, Boche I, Gille H, Brinkmann U: Development of secreted proteins as biotherapeutic agents. Expert Opin Biol Ther 2004, 4:551-8.

4. Hotez PJ, Zhan B, Bethony JM, Loukas A, Williamson A, Goud GN, Hawdon JM, Dobardzic A, Dobardzic R, Ghosh K, Bottazzi ME, Mendez S, 
Zook B, Wang Y, Liu S, Essiet-Gibson I, Chung-Debose S, Xiao S, Knox D, Meagher M, Inan M, Correa-Oliveira R, Vilk P, Shepherd HR, Brandt W, Russell PK: Progress in the development of a recombinant vaccine for human hookworm disease: the Human Hookworm Vaccine Initiative. Int J Parasitol 2003, 33:1245-1258.

5. Hewitson JP, Grainger JR, Maizels RM: Helminth immunoregulation: the role of parasite secreted proteins in modulating host immunity. $\mathrm{Mol}$ Biochem Parasitol 2009, 167:1-11.

6. Simpson JT, Wong K, Jackman SD, Schein JE, Jones SJ, Birol I: ABySS: a parallel assembler for short read sequence data. Genome Res 2009, 19:1117-1123.

7. Zerbino DR, Birney E: Velvet: algorithms for de novo short read assembly using de Bruijn graphs. Genome Res 2008, 18:821-829.

8. Chevreux B, Pfisterer T, Drescher B, Driesel AJ, Muller WE, Wetter T, Suhai S: Using the miraEST assembler for reliable and automated mRNA transcript assembly and SNP detection in sequenced ESTs. Genome Res 2004, 14:1147-1159.

9. Miller JR, Koren S, Sutton G: Assembly algorithms for next-generation sequencing data. Genomics 2010, 95:315-327.

10. Kumar S, Blaxter ML: Comparing de novo assemblers for 454 transcriptome data. BMC Genomics 2010, 11:571

11. Young ND, Jex AR, Cantacessi C, Hall RS, Campbell BE, Spithill TW Tangkawattana S, Tangkawattana P, Laha T, Gasser RB: A portrait of the transcriptome of the neglected trematode, Fasciola gigantica-biological and biotechnological implications. PLOS Negl Trop Dis 2011, 5:e1004.

12. Young ND, Hall RS, Jex AR, Cantacessi C, Gasser RB: Elucidating the transcriptome of Fasciola hepatica - a key to fundamental and biotechnological discoveries for a neglected parasite. Biotechnol Adv 2010, 28:222-231.

13. Cantacessi $C$, Mitreva M, Campbell BE, Hall RS, Young ND, Jex AR, Ranganathan S, Gasser RB: First transcriptomic analysis of the economically important parasitic nematode, Trichostrongylus colubriformis, using a next-generation sequencing approach. Infect Genet Evol 2010, 10:1199-1207.

14. Cantacessi C, Jex AR, Hall RS, Young ND, Campbell BE, Joachim A, Nolan MJ, Abubucker S, Sternberg PW, Ranganathan S, et al: A practical, bioinformatic workflow system for large data sets generated by nextgeneration sequencing. Nucleic Acids Res 2010, 38:e171.

15. Cantacessi C, Campbell BE, Young ND, Jex AR, Hall RS, Presidente PJ, Zawadzki JL, Zhong W, Aleman-Meza B, Loukas A, et al: Differences in transcription between free-living and $\mathrm{CO} 2$-activated third-stage larvae of Haemonchus contortus. BMC Genomics 2010, 11:266.

16. Cantacessi C, Gasser RB, Strube C, Schnieder T, Jex AR, Hall RS, Campbell BE, Young ND, Ranganathan S, Sternberg PW, Mitreva M: Deep insights into Dictyocaulus viviparus transcriptomes provides unique prospects for new drug targets and disease intervention. Biotechnol Adv 2011, 29:261-271.

17. Cantacessi C, Mitreva M, Jex AR, Young ND, Campbell BE, Hall RS, Doyle MA, Ralph SA, Rabelo EM, Ranganathan S, et al: Massively paralle sequencing and analysis of the Necator americanus transcriptome. PLoS Negl Trop Dis 2010, 4:e684.

18. Young ND, Campbell BE, Hall RS, Jex AR, Cantacessi C, Laha T, Sohn WM Sripa B, Loukas A, Brindley PJ, Gasser RB: Unlocking the transcriptomes of two carcinogenic parasites, Clonorchis sinensis and Opisthorchis viverrini. PLoS Negl Trop Dis 2010, 4:e719.

19. Dicker AJ, Nath M, Yaga R, Nisbet AJ, Lainson FA, Gilleard JS, Skuce PJ: Teladorsagia circumcincta: the transcriptomic response of a multi-drug resistant isolate to ivermectin exposure in vitro. Exp Parasitol 2011, 127:351-356

20. Nickel W: The mystery of nonclassical protein secretion. A current view on cargo proteins and potential export routes. Eur J Biochem 2003, 270:2109-2119.

21. Bendtsen JD, Jensen LJ, Blom N, Von Heijne G, Brunak S: Feature-based prediction of non-classical and leaderless protein secretion. Protein Eng Des Sel 2004, 17:349-356.

22. Bennuru S, Semnani R, Meng Z, Ribeiro JM, Veenstra TD, Nutman TB: Brugia malayi excreted/secreted proteins at the host/parasite interface: stage- and gender-specific proteomic profiling. PLoS Neg/ Trop Dis 2009 3:e410.

23. Nagaraj SH, Gasser RB, Ranganathan S: Needles in the EST haystack: largescale identification and analysis of Excretory-Secretory (ES) proteins in parasitic nematodes using Expressed Sequence Tags (ESTs). PLoS Negl Trop Dis 2008, 2:e301.

24. Mao X, Cai T, Olyarchuk JG, Wei L: Automated genome annotation and pathway identification using the KEGG Orthology (KO) as a controlled vocabulary. Bioinformatics 2005, 21:3787-3793.

25. Wu J, Mao X, Cai T, Luo J, Wei L: KOBAS server: a web-based platform for automated annotation and pathway identification. Nucleic Acids Res 2006, 34:W720-724.

26. Moriya $Y$, Itoh M, Okuda S, Yoshizawa AC, Kanehisa M: KAAS: an automatic genome annotation and pathway reconstruction server. Nucleic Acids Res 2007, 35:W182-185.

27. Viney ME: The biology and genomics of Strongyloides. Med Microbiol Immunol 2006, 195:49-54.

28. Aguinaldo AM, Turbeville JM, Linford LS, Rivera MC, Garey JR, Raff RA, Lake JA: Evidence for a clade of nematodes, arthropods and other moulting animals. Nature 1997, 387:489-493.

29. The C. elegans Sequencing Consortium: Genome sequence of the nematode C. elegans: a platform for investigating biology. Science 1998, 282:2012-2018

30. Stein LD, Bao Z, Blasiar D, Blumenthal T, Brent MR, Chen N, Chinwalla A Clarke L, Clee C, Coghlan A, et al: The genome sequence of Caenorhabditis briggsae: a platform for comparative genomics. PLOS Biol 2003, 1.E45

31. Ghedin E, Wang S, Spiro D, Caler E, Zhao Q, Crabtree J, Allen JE, Delcher AL, Guiliano DB, Miranda-Saavedra D, et al: Draft genome of the filarial nematode parasite Brugia malayi. Science 2007, 317:1756-1760.

32. Surget-Groba Y, Montoya-Burgos Jl: Optimization of de novo transcriptome assembly from next-generation sequencing data. Genome Res 2010, 20:1432-1440.

33. Strongyloides ratti cDNA sequencing data. , Available at: http://worm1.liv. ac.uk/file_summary.html.

34. Mello LV, O'Meara H, Rigden DJ, Paterson S: Identification of novel aspartic proteases from Strongyloides ratti and characterisation of their evolutionary relationships, stage-specific expression and molecular structure. BMC Genomics 2009, 10:611

35. Sff extract software. Available at: http://bioinf.comav.upv.es/sff_extract.

36. Huang $X$, Madan A: CAP3: a DNA sequence assembly program. Genome Res 1999, 9:868-877.

37. Iseli C, Jongeneel CV, Bucher P: ESTScan: a program for detecting, evaluating, and reconstructing potential coding regions in EST sequences. Proc Int Conf Intell Syst Mol Biol 1999, 138-148.

38. Bendtsen JD, Nielsen $H$, von Heijne G, Brunak S: Improved prediction of signal peptides: SignalP 3.0. J Mol Biol 2004, 340:783-795.

39. Emanuelsson $\mathrm{O}$, Nielsen $\mathrm{H}$, Brunak $\mathrm{S}$, von Heijne G: Predicting subcellular localization of proteins based on their N-terminal amino acid sequence. $\mathrm{J}$ Mol Biol 2000, 300:1005-1016.

40. Krogh A, Larsson B, von Heijne G, Sonnhammer EL: Predicting transmembrane protein topology with a hidden Markov model: application to complete genomes. J Mol Biol 2001, 305:567-580.

41. Craig $H$, Wastling JM, Knox DP: A preliminary proteomic survey of the in vitro excretory/secretory products of fourth-stage larval and adult Teladorsagia circumcincta. Parasitology 2006, 132:535-543.

42. Gourbal BE, Guillou F, Mitta G, Sibille P, Theron A, Pointier JP, Coustau C: Excretory-secretory products of larval Fasciola hepatica investigated using a two-dimensional proteomic approach. Mol Biochem Parasitol 2008, 161:63-66.

43. Ju JW, Joo HN, Lee MR, Cho SH, Cheun HI, Kim JY, Lee YH, Lee KJ, Sohn WM, Kim DM, et al: Identification of a serodiagnostic antigen, legumain, by immunoproteomic analysis of excretory-secretory products of Clonorchis sinensis adult worms. Proteomics 2009, 9:3066-3078.

44. Knudsen GM, Medzihradszky KF, Lim KC, Hansell E, McKerrow JH: Proteomic analysis of Schistosoma mansoni cercarial secretions. Mol Cell Proteomics 2005, 4:1862-1875.

45. Liu F, Cui SJ, Hu W, Feng Z, Wang ZQ, Han ZG: Excretory/secretory proteome of the adult developmental stage of human blood fluke Schistosoma japonicum. Mol Cell Proteomics 2009, 8:1236-1251.

46. Moreno Y, Geary TG: Stage- and gender-specific proteomic analysis of Brugia malayi excretory-secretory products. PLoS Negl Trop Dis 2008, 2 e326.

47. Smith SK, Nisbet AJ, Meikle LI, Inglis NF, Sales J, Beynon RJ, Matthews JB: Proteomic analysis of excretory/secretory products released by 
Teladorsagia circumcincta larvae early post-infection. Parasite Immunol 2009, 31:10-19.

48. Mulvenna J, Hamilton B, Nagaraj SH, Smyth D, Loukas A, Gorman JJ: Proteomics analysis of the excretory/secretory component of the bloodfeeding stage of the hookworm, Ancylostoma caninum. Mol Cell Proteomics 2009, 8:109-121.

49. Knudsen GM, Medzihradszky KF, Lim KC, Hansell E, McKerrow JH: Proteomic analysis of Schistosoma mansoni cercarial secretions. Mol Cell Proteomics 2005, 4:1862-1875.

50. Altschul SF, Gish W, Miller W, Myers EW, Lipman DJ: Basic local alignment search tool. J Mol Biol 1990, 215:403-410.

51. Zdobnov EM, Apweiler R: InterProScan-an integration platform for the signature-recognition methods in InterPro. Bioinformatics 2001, 17:847-848.

52. Kanehisa M, Goto S, Furumichi M, Tanabe M, Hirakawa M: KEGG for representation and analysis of molecular networks involving diseases and drugs. Nucleic Acids Res 2010, 38:D355-360.

53. Kanehisa M, Goto S, Hattori M, Aoki-Kinoshita KF, Itoh M, Kawashima S, Katayama T, Araki M, Hirakawa M: From genomics to chemical genomics: new developments in KEGG. Nucleic Acids Res 2006, 34:D354-357.

54. Kanehisa M, Goto S: KEGG: kyoto encyclopedia of genes and genomes. Nucleic Acids Res 2000, 28:27-30.

55. Wormpep database. http://www.sanger.ac.uk/Projects/C_elegans/ WORMBASE/current/wormpep.shtml, release wormpep224, date Mar 04, 2011.

56. Parkinson J, Blaxter M: SimiTri-visualizing similarity relationships for groups of sequences. Bioinformatics 2003, 19(3):390-395.

57. Abe T, Nawa Y, Yoshimura K: Protease resistant interleukin-3 stimulating components in excretory and secretory products from adult worms of Strongyloides ratti. J Helminthol 1992, 66:155-158.

58. Tazir Y, Steisslinger V, Soblik H, Younis AE, Beckmann S, Grevelding CG, Steen H, Brattig NW, Erttmann KD: Molecular and functional characterisation of the heat shock protein 10 of Strongyloides ratti. Mol Biochem Parasitol 2009, 168:149-157.

59. Mitreva M, McCarter JP, Martin J, Dante M, Wylie T, Chiapelli B, Pape D, Clifton SW, Nutman TB, Waterston RH: Comparative genomics of gene expression in the parasitic and free-living nematodes Strongyloides stercoralis and Caenorhabditis elegans. Genome Res 2004, 14:209-220.

60. Liotta F, Siekierka Jj: Apicomplexa, trypanosoma and parasitic nematode protein kinases as antiparasitic therapeutic targets. Curr Opin Investig Drugs 2010, 11:147-156.

61. Jiang J, Struhl G: Regulation of the Hedgehog and Wingless signalling pathways by the F-box/WD40-repeat protein Slimb. Nature 1998, 391:493-496.

62. Thompson FJ, Barker GL, Nolan T, Gems D, Viney ME: Transcript profiles of long- and short-lived adults implicate protein synthesis in evolved differences in ageing in the nematode Strongyloides ratti. Mech Ageing Dev 2009, 130:167-172.

63. Tazir $Y$, Steisslinger $\mathrm{V}$, Soblik H, Younis AE, Beckmann S, Grevelding CG, Steen H, Brattig NW, Erttmann KD: Molecular and functional characterisation of the heat shock protein 10 of Strongyloides ratti. Mol Biochem Parasitol 2009, 168:149-157.

64. Bellafiore S, Shen Z, Rosso MN, Abad P, Shih P, Briggs SP: Direct identification of the Meloidogyne incognita secretome reveals proteins with host cell reprogramming potential. PLoS Pathog 2008, 4:e1000192.

65. Gomez Gallego S, Loukas A, Slade RW, Neva FA, Varatharajalu R, Nutman TB, Brindley PJ: Identification of an astacin-like metallo-proteinase transcript from the infective larvae of Strongyloides stercoralis. Parasitol Int 2005, 54:123-133.

66. Lun HM, Mak CH, Ko RC: Characterization and cloning of metalloproteinase in the excretory/secretory products of the infective-stage larva of Trichinella spiralis. Parasitol Res 2003, 90:27-37.

67. Yoshida A, Nagayasu E, Nishimaki A, Sawaguchi A, Yanagawa S, Maruyama H: Transcripts analysis of infective larvae of an intestinal nematode, Strongyloides venezuelensis. Parasitol Int 2011, 60:75-83.

68. Thompson FJ, Barker GL, Hughes L, Viney ME: Genes important in the parasitic life of the nematode Strongyloides ratti. Mol Biochem Parasitol 2008, 158:112-119.

69. Welcome Trust Sanger Institute: five year plan for helminth sequencing. , Available at: http://www.sanger.ac.uk/Projects/Helminths/.
doi:10.1186/1471-2164-12-S3-S14

Cite this article as: Garg and Ranganathan: In silico secretome analysis approach for next generation sequencing transcriptomic data. $B M C$ Genomics 2011 12(Suppl 3):S14.

\section{Submit your next manuscript to BioMed Central and take full advantage of:}

- Convenient online submission

- Thorough peer review

- No space constraints or color figure charges

- Immediate publication on acceptance

- Inclusion in PubMed, CAS, Scopus and Google Scholar

- Research which is freely available for redistribution

Submit your manuscript at www.biomedcentral.com/submit
Ciomed Central 